\title{
Hubble Space Telescope/NICMOS Observations of Terzan 5: Stellar Content and Structure of the Core
}

\section{Citation}

Cohn, Haldan N., Phyllis M. Lugger, Jonathan E. Grindlay, and Peter D. Edmonds. 2002. "Hubble Space Telescope/NICMOS Observations of Terzan 5: Stellar Content and Structure of the Core." The Astrophysical Journal 571 (2): 818-29. https://doi.org/10.1086/339874.

\section{Permanent link}

http://nrs.harvard.edu/urn-3:HUL.InstRepos:41399882

\section{Terms of Use}

This article was downloaded from Harvard University's DASH repository, and is made available under the terms and conditions applicable to Other Posted Material, as set forth at http:// nrs.harvard.edu/urn-3:HUL.InstRepos:dash.current.terms-of-use\#LAA

\section{Share Your Story}

The Harvard community has made this article openly available.

Please share how this access benefits you. Submit a story.

Accessibility 


\title{
HUBBLE SPACE TELESCOPE/NICMOS OBSERVATIONS OF TERZAN 5: STELLAR CONTENT AND STRUCTURE OF THE CORE
}

\author{
Haldan N. Cohn and Phyllis M. Lugger \\ Department of Astronomy, Indiana University, 727 East Third Street, Bloomington, IN 47405; cohn@indiana.edu \\ AND \\ Jonathan E. Grindlay and Peter D. Edmonds \\ Harvard College Observatory, 60 Garden Street, Cambridge, MA 02138 \\ Received 2001 February 9; accepted 2002 January 28
}

\begin{abstract}
We report results from Hubble Space Telescope/NICMOS imaging of the extremely dense core of the globular cluster Terzan 5 . This highly obscured bulge cluster contains a low-mass X-ray binary and at least two millisecond pulsars, with evidence for a large millisecond pulsar population. It has been estimated to have one of the highest collision rates of any Galactic globular cluster, making its core a particularly conducive environment for the production of interacting binary systems. We have reconstructed high-resolution images of the central $19^{\prime \prime} \times 19^{\prime \prime}$ region of Terzan 5 by application of the drizzle algorithm to dithered NIC2 images in the F110W, F187W, and F187N near-infrared filters. We have used a DAOPHOT/ALLSTAR analysis of these images to produce the deepest color-magnitude diagram (CMD) yet obtained for the core of Terzan 5. We have also analyzed the parallel $11^{\prime \prime} \times 11^{\prime \prime} \mathrm{NIC} 1$ field, centered $30^{\prime \prime}$ from the cluster center and imaged in F110W and F160W, and an additional NIC2 field that is immediately adjacent to the central field. This imaging results in a clean detection of the red giant branch and horizontal branch in the central NIC2 field, and the detection of these plus the main-sequence turnoff and the upper main sequence in the NIC1 field. We have constructed an $H$ versus $J-H$ CMD for the NIC1 field, which provides a measurement of the infrared reddening, $E(J-H)=0.72$ [corresponding to $E(B-V)=2.16$ ]. We obtain a new distance estimate of $8.7 \mathrm{kpc}$, which places Terzan 5 within less than $1 \mathrm{kpc}$ of the Galactic center. The NIC1 CMD provides an apparent detection of the red giant branch bump, about 1 mag below the horizontal branch, indicating that the metallicity is at least solar and possibly somewhat higher. A number of blue objects are detected in the central NIC2 field and the NIC1 field, which hint at the presence of a blue horizontal branch. We have also determined a central surface-density profile that results in a maximum likelihood estimate of 7".9 \pm 0 ".6 for the cluster core radius. We discuss the implications of these results for the dynamical state of Terzan 5.
\end{abstract}

Subject headings: globular clusters: individual (Terzan 5) — Hertzsprung-Russell diagram infrared: stars

\section{INTRODUCTION}

The extremely dense core of the bulge globular cluster Terzan 5 makes it a likely location for the dynamical production of interacting binaries. It has been estimated to have the highest stellar interaction rate of any Galactic globular cluster (Verbunt \& Hut 1987). Indeed, Terzan 5 is one of only 12 clusters known to contain a low-mass X-ray binary (LMXB; Johnston, Verbunt, \& Hasinger 1995); this object exhibits both transient behavior and bursts. Terzan 5 also contains an eclipsing millisecond pulsar (MSP; Nice \& Thorsett 1992), many steep-spectrum radio point sources within $30^{\prime \prime}$ of the cluster center, and steep-spectrum extended radio emission in the cluster core (Fruchter \& Goss 1995). Lyne et al. (2000) have recently detected a second MSP just $10^{\prime \prime}$ from the cluster center. Based on the unresolved radio flux from the core of Terzan 5, Fruchter \& Goss (2000) argue that it may have the largest pulsar population of any cluster in the Galaxy. They also detect three discrete radio sources that they interpret as likely pulsars.

It has long been thought that dynamical interactions among stars in dense environments may lead to the production of interacting binaries and/or merged stars. Interaction mechanisms include two-body tidal capture and various three-body interactions, such as exchange reactions in which a massive degenerate star may replace one member of a main-sequence binary. This picture is supported by $H u b$ ble Space Telescope (HST) studies, which have made the first detections of cataclysmic variables $(\mathrm{CVs})$ in cluster cores (e.g., in NGC 6397; Cool et al. 1995) and have also detected substantial, centrally concentrated blue straggler populations (e.g., in M80; Ferraro et al. 1999b).

Ground-based study of Terzan 5 has been hampered by its high obscuration $\left(A_{V} \approx 7.7\right.$; Ortolani, Barbuy, \& Bica 1996) and the extreme crowding in its core. Given its Galactic coordinates, $(l=3.8, b=1.7)$, the line of sight to Terzan 5 passes within $0.6 \mathrm{kpc}$ of the Galactic center. Ortolani et al. (1996) produced the first color-magnitude diagram (CMD) for Terzan 5, from imaging obtained under conditions of exceptional seeing $(0.3-0.15)$ at the New Technology Telescope. Nevertheless, their $I$ versus $(V-I)$ CMD reaches only to just below the horizontal branch (HB) in the central 0.5 radius about the cluster center. Moreover, their CMD is strongly affected by differential reddening, with the red giant branch (RGB) spanning nearly a magnitude in color. Based on their CMD, Ortolani et al. (1996) estimate that the metallicity of Terzan 5 is approximately solar, making it one of the most metal-rich of all globular clusters. This is consistent with the range of earlier estimates of $[\mathrm{Fe} / \mathrm{H}]=+0.24$, based on integrated infrared photometry (Zinn 1985, hereafter Z85), $[\mathrm{Fe} / \mathrm{H}]=-0.28$, based on integrated spectroscopy (Armandroff \& Zinn 1988), and 
$[\mathrm{Fe} / \mathrm{H}]=+0.07$, also based on integrated spectroscopy (Bica et al. 1998). Ortolani et al. (1996) also obtained a distance estimate of $d=5.6 \mathrm{kpc}$ from their CMD, suggesting that Terzan 5 may be significantly closer than previous estimates, which generally lie in the range $d=7-9 \mathrm{kpc}$. Given its Galactic coordinates, this range allows the possibility that Terzan 5 is located very close to the Galactic center.

Terzan 5 is a natural target for $H S T / \mathrm{NICMOS}^{1}$ imaging studies, both to better characterize the stellar content and structure of the central regions, and to search for additional evidence of the presence of interacting binaries there. We report here results from NICMOS observations of the central region of Terzan 5 . We describe the observations in $\S 2$, present the analysis in $\S 3$, and discuss our conclusions in $\S 4$. This paper concentrates on reporting the analysis of the cluster CMD and central structure. A companion paper reports the results of a search for variable stars and the counterparts of the bright LMXB and the eclipsing MSP (Edmonds et al. 2001). While the present paper was in the final stages of the refereeing process, an independent NICMOS study of the CMDs of several reddened bulge clusters, including Terzan 5, appeared in print (Ortolani et al. 2001). Their study focuses on cluster age determination by isophote fitting; we compare our results for the age of Terzan 5 with theirs in $\S 4.4$.

\section{OBSERVATIONS}

The primary targets for our NICMOS observations were the central region of Terzan 5 and the MSP position 0.5 from the center (Edmonds et al. 2001). We imaged Terzan 5 with two primary pointings, one in which NIC2 was centered on the cluster center and one in which NIC1 was centered on the MSP position. These observations produced parallel NIC1 observations at $30^{\prime \prime}$ from the cluster center and parallel NIC2 observations of a field adjacent to and slightly overlapping with the central field. Figure 1 shows the three fields used in the present study, viz., the central and adjacent NIC2 fields, and the NIC1 field that was obtained in parallel with the central field. The exposures used in this study include (1) eight exposures of the central NIC2 field in each of the filters F110W, F187W, and F187N, (2) eight exposures of the NIC1 field in each of the filters F110W, F160W, and F187N, and (3) two exposures of the offset NIC2 field in F187W. For the exposures of the NIC1 and central NIC2 fields, the durations were $320 \mathrm{~s}$ for the broadband (W) filters and $1536 \mathrm{~s}$ for the narrowband (N) filter. For the offset NIC2 field, the total exposure time was $832 \mathrm{~s}$.

The eight observations of the central NIC2 field and the parallel NIC1 field were dithered, in order to increase the effective angular resolution and, consequently, the photometric accuracy in this extremely crowded field. The F187W and F187N filters, which are centered on Paschen- $\alpha$, were chosen for identifying emission-line objects. The F110W filter (which approximately corresponds to the Johnson $J$ band) was included to produce a broadband CMD, in combination with the F187W filter. Since F187W is not available for NIC1, we instead used F160W (which approximately corresponds to the Johnson $H$ band) for the redder broadband filter for this camera. While this has the

${ }^{1}$ NICMOS: Near-Infrared Camera and Multi-Object Spectrometer

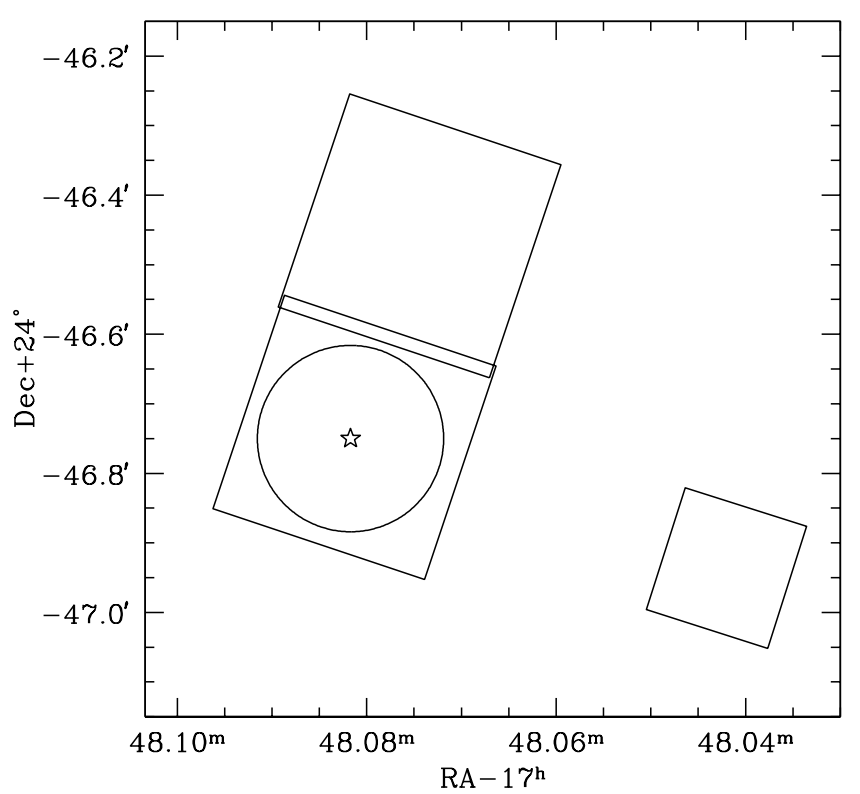

FIG. 1.- Map of the three NICMOS fields used in this study. North is up, and east is to the left. The star indicates our determined center of Terzan 5 , and the circle indicates our maximum likelihood determination of the core radius. The two eastern fields were observed with the NIC2 (field width $=19$ !'2), while the smaller western field was observed with the NIC1 (field width $\left.=11^{\prime \prime}\right)$.

disadvantage of providing a somewhat different CMD for the NIC1 field, a substantial advantage is that it allowed us to construct a NIC1-field CMD in a standard ground-based filter system $(J$ and $H)$. This proved to be quite valuable in allowing us to determine new values for the metallicity, reddening, and distance of Terzan 5 , as described below.

The eight dither positions for the NIC2 field were selected in a $3 \times 3$ array (minus one corner position). The basic step between dither positions was 3 NIC2 pixels $(0.23)$, with a random 0.125 NIC2 pixel (0".009) step added to provide subpixel sampling. Each dither position was observed for one HST orbit. The eight-orbit observation sequence was split over two visits, separated in time by 1 month. Cross correlations of the resulting images indicate that subpixel pointing was accurately maintained from orbit to orbit within each visit. However, there were pointing shifts by about 0.5 and 0.2 NIC2 pixels in the two orthogonal directions between the visits. These additional shifts acted in concert with the intentional 0.125 pixel shifts to provide subpixel sampling.

\section{DATA ANALYSIS}

\subsection{Drizzle Reconstruction of Images}

The extraordinarily crowded central NIC2 field presents a substantial challenge to photometric analysis. The NIC1 and NIC2 provide full Nyquist sampling (i.e., 2 pixels resolution per element) down to 1 and $1.7 \mu \mathrm{m}$, respectively. Thus, the NIC2 images in the F110W band are strongly undersampled. The effect of this undersampling is alleviated by our dithering strategy for the central region. In order to make optimal use of the information contained in the dithered images, we employed the "drizzle" (variable-pixel linear reconstruction) algorithm of Hook \& Fruchter (1997) to recover the full resolution provided by the HST optics. We 
used the implementation of this algorithm from the STSDAS extensions of IRAF, ${ }^{2}$ as described by Hook, Pirzkal, \& Fruchter (1999).

For the reconstructed NIC1 and NIC2 images, we chose pixel sizes of $0 . \prime 022$ and $0 . \prime 038$, respectively, which are half of the original pixel sizes. We chose a value of 0.5 input pixel for the "drop" size; this parameter determines the magnification of the projection of each input pixel into the output image. Examination of the output drizzled images indicated a moderate level of residual pixel-to-pixel fluctuation, suggesting incomplete point-spread function (PSF) sampling. These fluctuations were particularly evident in the vicinity of stellar image cores in the residual image on performing PSF subtraction photometry. We reduced this noise by applying a Gaussian smoothing filter $(\sigma=0.75$ pix $)$ to the drizzled images. Figure 2 shows a comparison of a single F110W exposure of the central region and the result of combining the eight F110W images using the drizzle algorithm.

\subsection{Photometry}

Given the substantial increase in the resolution of the PSF that results from the application of the drizzle algorithm, it is feasible to carry out PSF subtraction photometry. We employed the DAOPHOT/ALLSTAR software package (Stetson 1987; Stetson \& Harris 1988) to determine an empirical PSF for each frame and simultaneously fit this to all detected stars. Approximately 100-150 stars were used to determine a PSF with quadratic spatial variation across the frame. A complication in the process lies in distinguish-

2 IRAF is distributed by the National Optical Astronomy Observatories, which are operated by the Association of Universities for Research in Astronomy, Inc., under cooperative agreement with the National Science Foundation. ing between faint stars and artifacts of the complex PSF structure of bright stars. We used visual inspection of apparent detections in the vicinity of bright stars to help filter out artifacts. Since the diffraction pattern has a strong wavelength dependence, intercomparison of the F110W and F160W images proved useful in this filtering process. A PSF radius of 20 pixels was used for both the NIC1 and NIC2 fields. This size was chosen as a compromise between the goals of subtracting as much of the halos of bright stars as possible, while still retaining a sufficient number of stars, with no brighter neighbors within the PSF radius, to accurately characterize the spatial variation of the PSF.

Given the higher degree of crowding in the central NIC2 field, relative to the offset NIC1 field, and the nearly twice as large NIC2 pixels, PSF subtraction was less effective in the NIC2 field. A clear indication of this is that the limiting magnitude is considerably fainter in the NIC1 field for the same amount of exposure (see $\S 4$ ). Also, examination of the residual frames for the NIC1 and NIC2 fields indicates a considerably greater amount of residual light in the latter. Nevertheless, PSF subtraction photometry produced significantly better results than simple aperture photometry, for both the NIC1 and NIC2 frames.

\subsection{Calibration}

The NICMOS pipeline calibration system produces images that are calibrated in flux units; i.e., the images are given in count rate (data numbers per second). These can be converted to physical flux units (janskys or ergs s${ }^{-1} \mathrm{~cm}^{-2}$ $\AA^{-1}$ ) by multiplying by a corresponding scale factor that is tabulated for each filter. These fluxes can then be expressed in magnitude units in the $\mathrm{AB}_{\nu}$ or Vega systems; we use the latter. As noted previously, the F110W and F160W filters, which were used for the NIC1 field, are analogs to the ground-based $J$ and $H$ bands. We computed a linear trans-

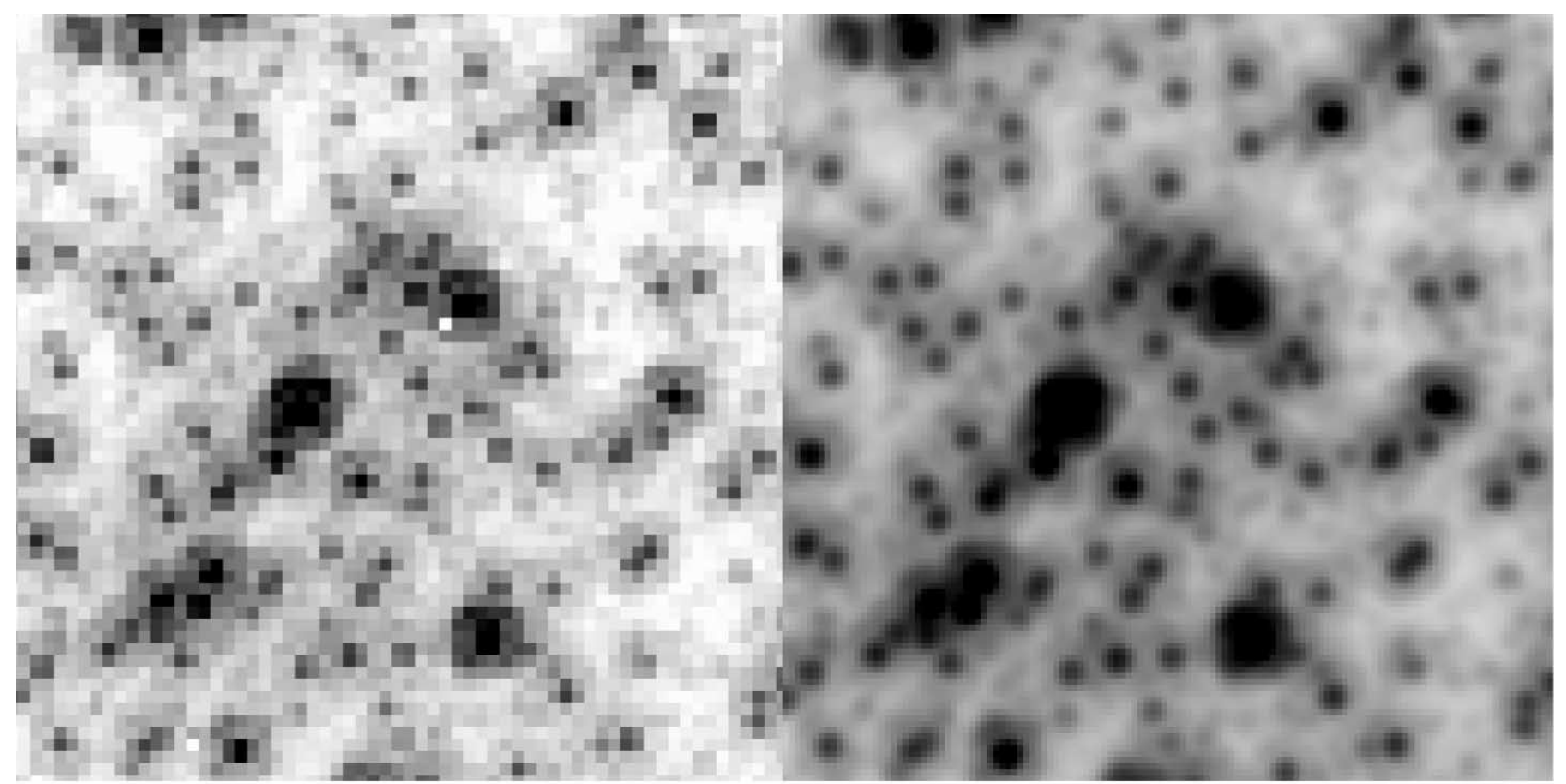

FIG. 2.-Comparison of the central $5^{\prime \prime} \times 5^{\prime \prime}$ section of a single F110W NIC2 exposure of the cluster center (left) with the corresponding section of the image that results from a drizzle reconstruction from eight dithered images (right). 
formation between the HST and ground-based systems using NICMOS photometry for five standard stars with a wide color range $(-0.1 \leq J-H \leq+2.1)$ that are listed in the NICMOS Data Handbook, version 4.0. Preliminary NICMOS photometry for the stars is given at the NICMOS web site. The resulting transformations are

$$
\begin{gathered}
J=\mathrm{F} 110 \mathrm{~W}+0.033-0.335(\mathrm{~F} 110 \mathrm{~W}-\mathrm{F} 160 \mathrm{~W}), \\
H=\mathrm{F} 160 \mathrm{~W}-0.001-0.092(\mathrm{~F} 110 \mathrm{~W}-\mathrm{F} 160 \mathrm{~W}) .
\end{gathered}
$$

These results are almost identical to those of Schulte-Ladbeck et al. (1999), who followed a similar calibration procedure. The rms residual is 0.16 mag for the $J$-transformation and $0.05 \mathrm{mag}$ for the $H$-transformation. The large value of the former is mostly determined by one large residual (for the red standard BRI0021 with $J-H=1.17$ ); the rms of the other four $J$-residuals is $0.07 \mathrm{mag}$. We also note the existence of a significant color term in the $J$-transformation, which also contributes to the overall uncertainty.

The transformations given by equations (1) and (2) allowed us to construct a standard near-infrared CMD for the NIC1 field. Since F187W (rather than F160W) serves as the redder broadband filter in the central NIC2 field, it is necessary to use a color index for this field that does not have a strict ground-based analog.

\section{THE COLOR-MAGNITUDE DIAGRAM}

In constructing CMDs, we required that the centroids of stars determined by ALLSTAR match to 0.5 pixel between the F110W and F187W frames. This tight limit on the positional offset helped to filter out both weakly detected stars with uncertain photometry and PSF artifacts. We also eliminated stars that have neighbors with comparable magnitudes and centroids within 3 pixels, since these were strongly blended and thus produced substantial photometric uncertainty.

Figure 3 shows a comparison of the CMDs obtained for the NIC2 and NIC1 fields. The ordinate is F110W in both cases. It is immediately obvious that the central NIC2 field is much more populous, owing to the much higher stellar density and the 4 times larger area. Not surprisingly, the offset NIC1 field produces much deeper photometry, given the lower crowding and the smaller pixel size. The horizontal branch $(\mathrm{HB})$ is clearly detected in both fields at a magnitude of F110W $\approx 15.9$, demonstrating the consistency of the calibration between the NIC1 and NIC2 cameras. The NIC1 photometry also clearly detects the main-sequence turnoff (MSTO) at $\mathrm{F} 110 \mathrm{~W} \approx 20$ and upper main sequence; the faint-end limit of the NIC2 photometry is brighter than this at $\mathrm{F} 110 \mathrm{~W} \approx 19$.

While one of the original goals of this study was to reach below the MSTO in the central NIC2 field, in order to survey the core of Terzan 5 for CVs, this is clearly not possible. Even with extensive dithering, the extraordinary crowding of this region substantially limits the accuracy of the photometry, particularly for F110W $>18$, which is just 2 mag below the HB. Nevertheless, the photometry in the central NIC2 field substantially supersedes the quality of the excellent ground-based $V$ and $I$ photometry reported by Ortolani et al. (1996). Examination of their CMD for the central $26^{\prime \prime}$ radius region indicates that the limiting magnitude is not much more than 1 mag below the HB and that

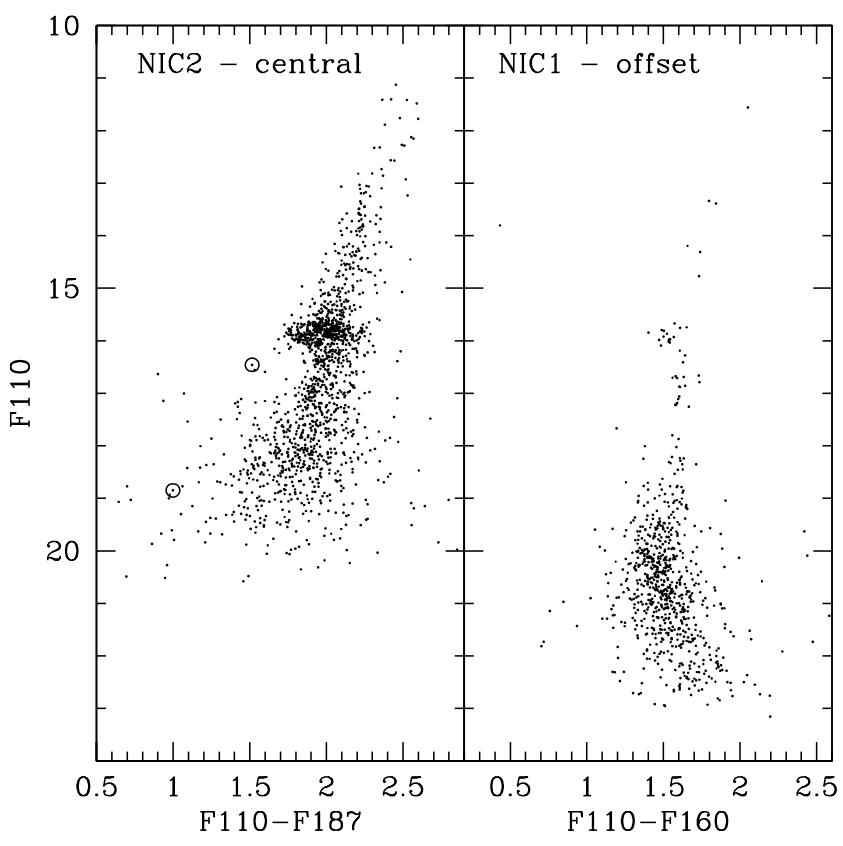

FIG. 3.-Comparison of the CMDs for the central NIC2 field (left $)$ and the NIC1 field (right). The ordinate is F110W in both cases, while the abscissa is the available broadband color index. The much greater depth of the NIC1 CMD is a consequence of the much lower crowding and 2 times higher resolution in this field. The circled objects in the NIC2 field are photometrically variable (Edmonds et al. 2001); the brighter object is an RRab Lyrae, and the fainter one is likely an eclipsing blue straggler.

the width of the RGB is $\Delta(V-I)=0.92$; they ascribe this to differential reddening. The greatly increased depth and precision of the CMD that is afforded by our NICMOS observations permits us to search for unusual blue objects below the HB, if not below the MSTO. Furthermore, as has been recently demonstrated by Davidge (2000, 2001), near-infrared CMDs provide valuable information on the metallicities, reddenings, and distances of low-latitude globular clusters.

In addition to constructing broadband CMDs, we also constructed an F187N-F187W versus F187W diagram to search for evidence of emission-line objects. We find no convincing detections of such objects in either the central NIC2 field or the NIC1 field, in agreement with the analysis of the same data set by Edmonds et al. (2001, see their Figure 8b).

\subsection{Blue Stars below the $H B$}

Figure 3 indicates a number of stars that lie blueward of the RGB in the NIC2 field. Edmonds et al. (2001) have shown that two of these blue stars show significant variability; these are circled in the left panel of Figure 3. As discussed by Edmonds et al. (2001), the brighter of these variables is an RRab Lyrae, while the fainter is likely an eclipsing blue straggler or possibly the LMXB counterpart. These two stars are both located at $8^{\prime \prime}$ from the cluster center. This puts the objects at the projected core radius. While the possibility of a chance superposition always exists, particularly in a crowded field, the small radial offset of these stars from the cluster center makes a superposition of a nonmember unlikely. Moreover, given the consistency of the apparent magnitude of the RR Lyrae with the value expected for one located in Terzan 5, it appears likely that the star is at the cluster distance. While the photometry of 
the RR Lyrae appears fairly secure, the candidate blue straggler is clearly in the region where photometric uncertainty substantially widens the RGB. No variability is observed from the other two stars that lie close to the RR Lyrae in the CMD.

Given the extreme crowding of the central field of Terzan 5 , imperfect PSF-subtraction photometry can produce large photometric error as well as outright artifacts, e.g., PSF "tendrils" that are detected as stars. We performed several tests of the photometry of the apparently blue stars, including visual inspection of the detections in the drizzled frames, variations in the photometric procedure, and cuts on the magnitude error estimates provided by ALLSTAR. Visual inspections generally proved useful at and above the HB level, where photometric problems were fairly clear. However, by about 1 mag below the HB, it became quite difficult to discern photometric problems in this way, owing to the extreme crowding of the field and the complexity of the PSF. Thus, we deemed automated photometric tests to be more useful for faint stars. To this end, we investigated cuts on the formal magnitude error estimates provided by ALLSTAR. The result of one such experiment is shown in Figure 4 . In this case, a limit of 0.03 was placed on the formal errors of the F110W and F187W magnitudes. The three bluest stars are most likely foreground objects; a strong sequence of such objects is seen in the $2.2 \times 2.2$ field surveyed by Ortolani et al. (1996).

Figure 4 provides a hint of the presence of a blue horizontal branch (BHB) that is parallel to the RGB and about 0.5 mag bluer in color. This is the expected location of the BHB in infrared colors, as can be seen in the photometry of four metal-poor clusters by Davidge \& Courteau (1999). The hint of a BHB in Terzan 5 is intriguing, since the presence of a $\mathrm{BHB}$ in an extremely metal-rich cluster is unusual. Rich et

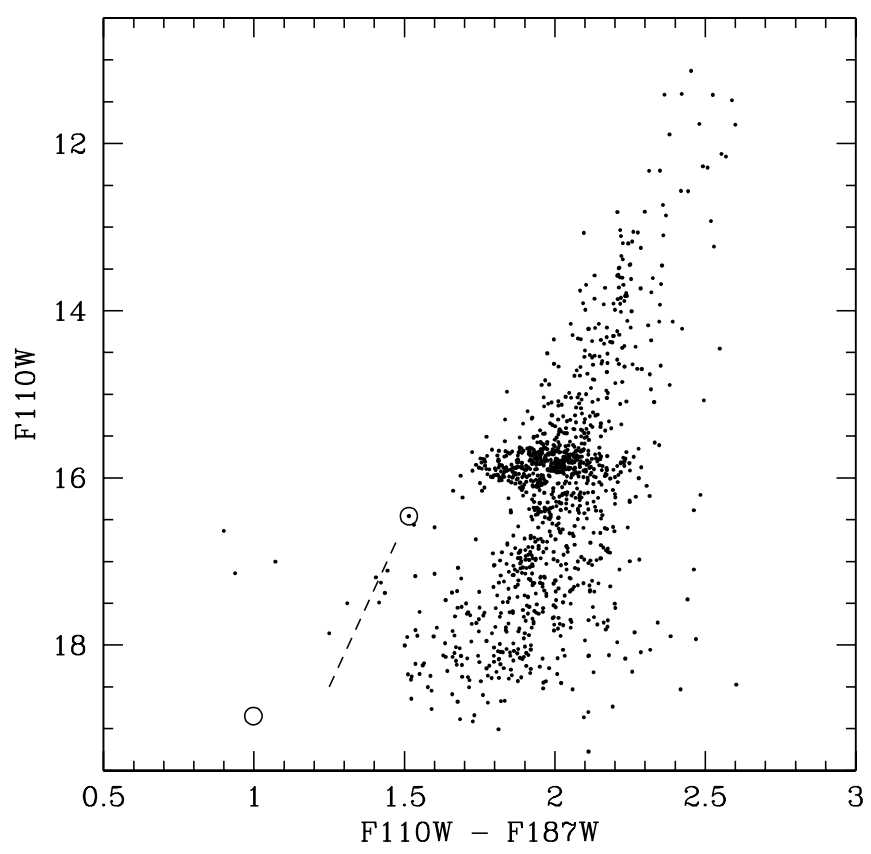

FIG. 4. - CMD for the central NIC2 field. The photometry is the same as in the left panel of Fig. 3, with a 0.03 mag limit placed on the formal photometric error. The circled objects are the variables noted in Fig. 3; the fainter one does not pass the magnitude error filter. The dashed line indicates the location of the possible blue horizontal branch. The three bluest stars near $\mathrm{F} 110 \mathrm{~W}=17$ are probably foreground stars. al. (1997) first discovered substantial BHB populations in old, metal-rich systems. They found a blue extension of the strong red horizontal branch (RHB) in the globular clusters NGC 6388 and NGC 6441, based on HST WFPC2 BV imaging. These clusters have high $[\mathrm{Fe} / \mathrm{H}]$ values of -0.60 and -0.53 , respectively. Both clusters also have extremely high central densities, with $\log \rho_{0}\left(M_{\odot} \mathrm{pc}^{-3}\right)$ values of 5.3 and 5.4, respectively (Djorgovski 1993). Rich et al. (1997) suggest that the presence of a BHB in these clusters may be the result of enhanced mass loss from giants induced by stellar interactions. However, they note that the $\mathrm{BHB} / \mathrm{RHB}$ ratio does not show the radial gradient that might be expected if $\mathrm{BHB}$ stars were produced by dynamical interactions in the cluster cores. Moreover, Bedin et al. (2000) have shown that the extended BHB population in the cluster NGC 2808 is no more centrally concentrated than the RGB population. A complicating factor is that once a star loses a substantial amount of envelope mass, by either stellar interactions or normal giant winds, it will tend to move to larger orbital radius as a consequence of mass segregation. Given HB lifetimes of $\sim 10^{8} \mathrm{yr}$, this mass segregation process has enough time to at least partially reverse an initial central concentration of BHB stars.

Since Terzan 5 has an even higher central density $\left(\log \rho_{0}=5.8\right.$; Djorgovski 1993) than do NGC 6388 and NGC 6441, dynamical interaction effects should be even more important for Terzan 5. Given the handful of potential BHB stars detected by our photometry, it is not feasible to examine the radial dependence of the $\mathrm{BHB} / \mathrm{RHB}$ ratio. This will require wide-field infrared imaging of Terzan 5 .

\subsection{The RGB Bump and Metallicity}

Stellar evolution theory predicts the presence of an RGB "bump," a pileup that results from a pause in the ascent of the RGB that occurs when the hydrogen burning shell extends past the point of last maximum depth of the convective envelope (see, e.g., Iben 1968). Fusi Pecci et al. (1990) demonstrated that the location of the bump on the RGB is correlated with the cluster metallicity. Ferraro et al. (1999a) have measured the location of the RGB bump in 47 globular clusters and have calibrated the relation between the offset of the bump magnitude from the HB magnitude $\left(\Delta V_{\mathrm{HB}}^{\text {bump }} \equiv V_{\text {bump }}-V_{\mathrm{HB}}\right)$ and several different metallicity indices. The bump magnitude is brighter than the $\mathrm{HB}$ for metallicities below $[\mathrm{Fe} / \mathrm{H}] \approx-1.2$ and is fainter than the $\mathrm{HB}$ for higher metallicities. The clusters in the Ferraro et al. (1999a) survey range in $[\mathrm{Fe} / \mathrm{H}]$ from -2.2 to -0.2 . The high end of this range is of particular interest for comparison with Terzan 5 . The most metal-rich cluster in the sample is NGC 6528 with $[\mathrm{Fe} / \mathrm{H}]=-0.23$ on the Z85 scale and $\Delta V_{\mathrm{HB}}^{\mathrm{bump}}=+0.78$. Davidge (2000) has recently reported near-infrared photometry of NGC 6528, with the CanadaFrance-Hawaii Adaptive Optics system, and finds a similar bump offset in the infrared, viz., $\Delta K_{\mathrm{HB}}^{\text {bump }}=+0.6$.

Ferraro et al. (1999a) and Davidge (2000) note that examination of the cluster luminosity function provides a good means of determining the magnitude of the RGB bump. The top and middle panels of Figure 5 show F110W luminosity functions for the NIC2 and NIC1 fields, respectively. The results for the NIC2 frame are shown for both the entire frame and the corners of the frame only. The motivation for separately considering the corners is to reduce the influence of photometric error, which is greatest 


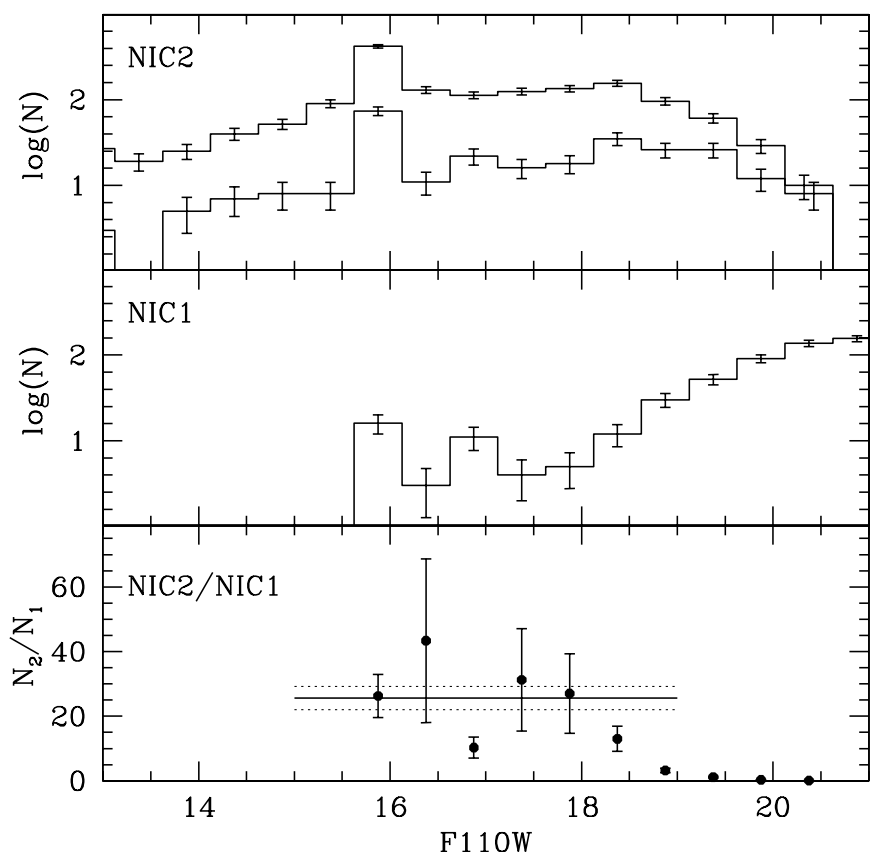

FIG. 5. - Comparison of the luminosity functions for the central NIC2 field (top) and the NIC1 field (middle). The two histograms for the NIC2 field represent the results for the entire frame (upper histogram) and the corners of the frame only (lower histogram). Here the "corners" are defined as the region outside of an inscribed circle of radius 9".6. The bottom panel shows the ratio of the luminosity functions for the entire NIC2 and NIC1 fields. The solid horizontal line is the overall count ratio for F110W $<18.4$, and the dotted horizontal lines represent the $1 \sigma$ errors on the ratio.

in the central part of the frame owing to the greater crowding there. The bottom panel shows the ratio of the NIC2 and NIC1 luminosity functions, which provides a measure of the completeness of the counts in the NIC2 field. Only bins containing at least three stars are shown. The solid horizontal line shows the overall count ratio for F110W $<18.4$. The NIC2/NIC1 count ratio shows that significant incompleteness sets in the NIC2 field for F110W > 18.0; this is about 2 mag fainter than the HB. This incompleteness is not surprising, given the extraordinarily high central density of Terzan 5, together with the complex NICMOS PSF structure.

The HB is clearly evident at F110W $=15.9$ in all of the histograms in Figure 5. There is also a smaller second peak at F110W $=16.9$ evident in the NIC1 histogram. This feature corresponds to the clump of stars seen below the HB in Figure 3, and appears to represent the RGB bump. To estimate its statistical significance, we compare its height (11 stars) to the mean of the two adjacent bins (three and four stars); the result is a $2.1 \sigma t$-ratio. Given that this is approximately where the RGB bump should occur in a very high metallicity cluster, we are fairly confident that we have detected it in the NIC1 field. In contrast, examination of the histogram for the entire NIC2 frame shows no evidence of an RGB bump. There is a hint of an RGB bump in the histogram for stars in the frame corners, although this feature has low statistical significance $(1.6 \sigma)$. It may be that a small RGB bump in the NIC2 field has been smeared out by photometric error. The lack of an RGB bump in the histogram for the entire NIC2 field is reflected by the dip in the NIC2/ $\mathrm{NIC} 1$ count ratio at $\mathrm{F} 110 \mathrm{~W}=16.9$.

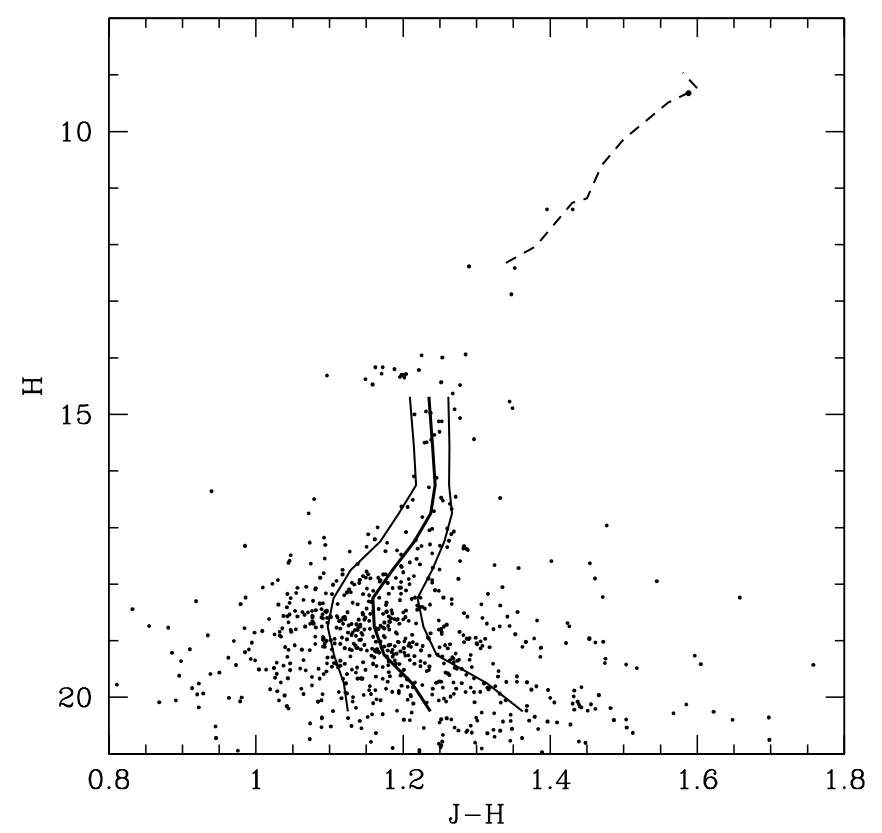

FIG. 6.-CMD for the offset NIC1 field. The field center is located at $30^{\prime \prime}$ from the cluster center. The photometry has been transformed to the $J H$ system. The dashed line is a fit of the Baade's window M giant sequence, with $E(J-H)=0.72$. The heavy solid curve is the CMD median ridge line, and the lighter solid curves represent the first and third quartiles of the color distribution.

The RGB bump detected in the NIC1 field provides an estimate of the metallicity of Terzan 5 . The middle panel of Figure 5 indicates a value of $\Delta \mathrm{F} 110 \mathrm{~W}_{\mathrm{HB}}^{\text {bump }}=+1.0$. We expect a similar value to hold for $\Delta V_{\mathrm{HB}}^{\mathrm{bump}}$, since there is little color difference between the RGB bump and the intersection of the HB with the RGB. As noted above, the largest $\Delta V_{\mathrm{HB}}^{\text {bump }}$ observed by Ferraro et al. (1999a) is +0.78 for NGC 6528. For $\Delta V_{\mathrm{HB}}^{\text {bump }}=+1.0$, their $\Delta V_{\mathrm{HB}}^{\mathrm{bump}}-[\mathrm{Fe} / \mathrm{H}]_{\mathrm{Z} 85}$ calibration gives $[\mathrm{Fe} / \mathrm{H}]_{\mathrm{Z} 85}=+0.26$ for Terzan 5 . This is in close agreement with the value of +0.24 from Z85, which is based on integrated infrared photometry. It is also consistent with the conclusion by Ortolani et al. (1996), based on the RGB curvature, that the metallicity of Terzan 5 is higher than that of NGC $6553\left([\mathrm{Fe} / \mathrm{H}]_{\mathrm{Z} 85}=-0.29\right)^{3}$ and is probably solar. Thus, there appears to be a strong indication that the metallicity of Terzan 5 is at least solar and is perhaps even somewhat higher. This is particularly interesting, in light of our detection of an RR Lyrae in the core (Edmonds et al. 2001; see $\S 4.1$ above), since this would make Terzan 5 the highest metallicity cluster in which an RR Lyrae has been observed. This appears to provide yet another indication of a high stellar interaction rate in the core of Terzan 5, on the assumption that collisionally induced mass loss was responsible for the production of the RR Lyrae.

\subsection{Reddening and Distance Modulus}

Figure 6 shows the CMD derived from the NIC1 frame photometry, calibrated in the Johnson $J H$ magnitude sys-

\footnotetext{
${ }^{3}$ Several recent determinations of the metallicity of NGC 6553 have been carried out by Barbuy et al. (1999), Cohen et al. (1999), and Coelho et al. (2001). While there are some differences among these studies in detail, there is general agreement that the metallicity of NGC 6553 is near solar.
} 
tem. The upper RGB is sparsely populated, given the small size of this field and its $30^{\prime \prime}$ offset from the cluster center. Nevertheless, the six stars above the HB are sufficient to characterize the location and slope of the upper RGB. The dashed line represents a reddened fit of the Baade's window M giant sequence from Frogel \& Whitford (1987), Table 3B, based on the Rieke \& Lebofsky (1985) infrared reddening law. The inferred infrared reddening is $E(J-H)=0.72$, and the total infrared extinction is $A_{H}=1.18$. This corresponds to a visual reddening of $E(B-V)=2.18$ and a $V$-band extinction of $A_{V}=6.75$. Our reddening estimate is somewhat smaller than the value of $E(B-V)=2.49$ obtained by Ortolani et al. (1996) from the position of the RGB in their ground-based VI CMD. Other previous reddening estimates include $E(B-V)=1.65$ (Armandroff \& Zinn 1988, based on a spectroscopic measure of an interstellar band), $E(B-V)=2.1$ (Malkan 1982, based on integrated infrared photometry), and $E(B-V)=2.42$ (Djorgovski 1993, method unspecified). Inasmuch as the properties of Terzan 5 are best determined in the infrared, it is interesting to note the close agreement between our new reddening estimate and that of Malkan (1982).

As we note in $\S 1$, Ortolani et al. (1996) obtained a significantly closer distance for Terzan $5(5.6 \mathrm{kpc})$ than have other investigators. If we simply replace their reddening value of $E(B-V)=2.49$ by our value of $E(B-V)=2.18$, their distance estimate would be increased to $8.7 \mathrm{kpc}$. Alternatively, we can estimate the distance by comparison with the highmetallicity cluster NGC 6528, which has been studied in the infrared by Davidge (2000). The reddening-corrected HB magnitude of NGC 6528 is $H_{\mathrm{HB}}=13.1$ versus 13.0 for Terzan 5. Adopting a distance of $9.1 \mathrm{kpc}$ for NGC 6528 (Harris $1996)^{4}$ also yields $d=8.7 \mathrm{kpc}$ for Terzan 5. Recent Galactic center distance determinations have produced values of $d_{\mathrm{GC}}=8.3 \pm 1.0 \mathrm{kpc}$ (Carney et al. 1995) and $d_{\mathrm{GC}}=$ $7.9 \pm 0.3 \mathrm{kpc}$ (McNamara et al. 2000). Since the line of sight to Terzan 5 passes within $0.6 \mathrm{kpc}$ of the Galactic center, the total distance of Terzan 5 from the Galactic center appears to be no more than about $1 \mathrm{kpc}$. Uncertainties in both the NICMOS calibration and the infrared reddening law contribute to the error in the distance determination. These uncertainties are not well quantified. We note that if the distance modulus is accurate to at least $0.4 \mathrm{mag}$, then the uncertainty in the distance is less than about $2 \mathrm{kpc}$.

The apparently small distance of Terzan 5 from the Galactic center is interesting in light of the metallicity gradient in the bulge predicted by dissipative collapse models for its formation (e.g., Mollá, Ferrini, \& Gozzi 2000). Frogel, Tiede, \& Kuchinski (1999) have measured a metallicity

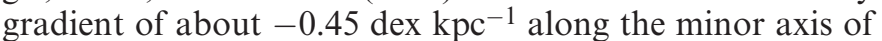
the bulge. Feltzing \& Gilmore (2000) have recently found a steeper gradient value of $-1.3 \mathrm{dex} \mathrm{kpc}^{-1}$. Based on nearinfrared spectroscopy of bulge giants, Ramírez et al. (2000) argue that there is little evidence for a gradient inside of 560 pc from the Galactic center and find a mean metallicity of $-0.21 \pm 0.30$ (s.d.) for the inner bulge. Since the metallicity that we find here for Terzan 5 is at least this large and the bulge metallicity drops outside of the inner bulge, an inner bulge origin for Terzan 5 appears to be a reasonable inference.

${ }^{4}$ See http://physun.physics.mcmaster.ca/Globular.html.

\subsection{The Main-Sequence Turnoff and Cluster Age}

As we noted in $\S 4$, it is evident from Figure 3 (right panel) that the NIC1-field photometry reaches below the MSTO. The narrow 1.0 mag color range used in the NIC1 JH CMD shown in Figure 6 emphasizes the spread of the points about the mean color-magnitude relation. To determine the location of the MSTO, we computed the median color for 0.5 mag bins in $H$. We also computed the first and third quartiles of the color distributions in each magnitude bin, in order to characterize the width of the distributions. These three color-distribution statistics are plotted as continuous curves in Figure 6. The median color reaches its bluest value near $H=18.5$, which is 4.3 mag below the HB. We identify this as the MSTO and note that its detection at $30^{\prime \prime}$ from the cluster center is an impressive demonstration of the capability of NIC1 imaging. The corresponding unreddened magnitude and color of the turnoff are $H_{\mathrm{TO}}=17.3$ and $(J-H)_{\mathrm{TO}}=0.4$. The semi-interquartile range of the color distribution at the turnoff is just $0.06 \mathrm{mag}$, indicating a fairly narrow photometric spread for a typical star.

The magnitude difference between the $\mathrm{HB}$ and the MSTO, $\Delta V_{\mathrm{HB}}^{\mathrm{TO}}$, is an important indicator of cluster age, as discussed by Chaboyer, Demarque, \& Sarajedini (1996). Girardi et al. (2000) have recently computed evolutionary tracks for low- to intermediate-mass stars with a wide metallicity range and have derived isochrones in the JohnsonCousins system, including the $J$ and $H$ bands. These can, in principle, be used to calibrate the relation between $\Delta H_{\mathrm{HB}}^{\mathrm{TO}}$ and cluster age. In applications of the $\Delta V_{\mathrm{HB}}^{\mathrm{TO}}$ method of age determination, it is usual to adopt a fixed absolute magnitude for the HB. This is typically the RR Lyrae magnitude, which is assumed to be independent of age over the relevant age range for globular clusters (see, e.g., Chaboyer, Demarque, \& Sarajedini 1996; Rosenberg et al. 1999). For very high metallicity clusters, where RR Lyrae stars may be deficient or absent, an alternative choice for defining a fixed $\mathrm{HB}$ magnitude, is the zero-age horizontal branch (ZAHB) magnitude for a star of a chosen fudicial mass and appropriate metallicity (see Ortolani et al. 2001, as discussed below).

As a cluster evolves, the magnitude of the ZAHB is expected to become somewhat fainter, as indicated by the Girardi et al. (2000) isochrones, which are based on evolutionary tracks that include $\mathrm{HB}$ and post-HB evolution. The resulting evolution of the red giant clump (i.e., red $\mathrm{HB}$ ) absolute magnitude is shown by Girardi \& Salaris (2001, see their Figure 1). Thus, an alternative procedure for calibrating the $\Delta H_{\mathrm{HB}}^{\mathrm{TO}}$-age relation is to use the evolving HB magnitude. In Figure 7, we compare these two calibration procedures for two values of the cluster metallicity. In the upper panel, we use the contemporaneous $\mathrm{HB}$ magnitude from the Girardi et al. (2000) isochrones, while in the lower panel we use the fixed, zero-age HB magnitude for a star with an initial mass of $1 M_{\odot}$, following Ortolani et al. (2001). We define the contemporaneous HB magnitude by the bluest point on the HB isochrones, since examination of the Girardi et al. (2000) isochrones indicates that stars linger near this location. It can be seen that the calibration curves flatten considerably for $t \gtrsim 10 \mathrm{Gyr}$ when the evolving HB magnitude is used. In both panels, we show the uncertainty range produced by an estimated $0.2 \mathrm{mag}$ precision in the determination of $\Delta H_{\mathrm{HB}}^{\mathrm{TO}}$.

The curves in the upper and lower panels of Figure 7 give similar results for the best-fit cluster age for a given value of 

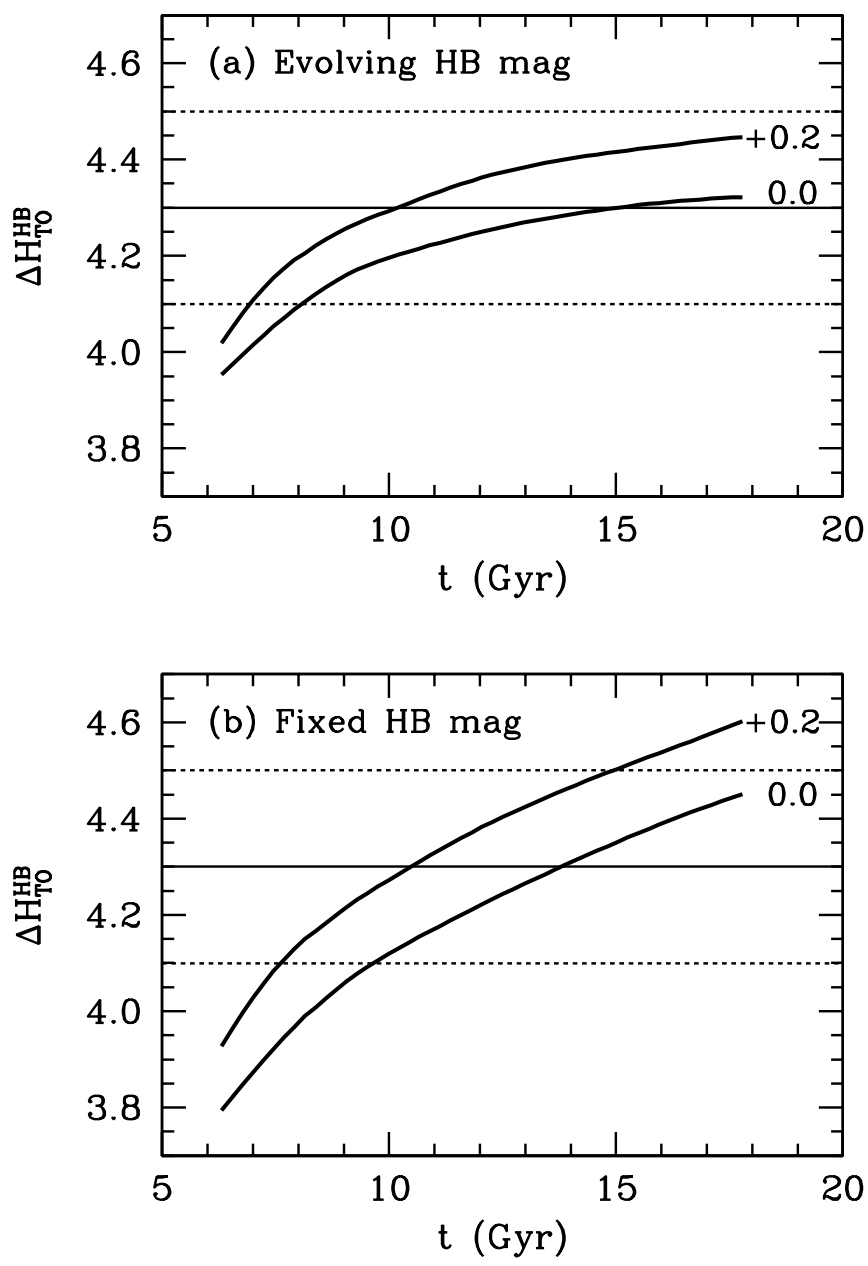

FIG. 7.-Magnitude difference between the horizontal branch and turnoff, $\Delta H_{\mathrm{HB}}^{\mathrm{TO}}$, vs. cluster age, for $(a)$ a time-evolving $\mathrm{HB}$ magnitude and $(b)$ a fixed $\mathrm{HB}$ magnitude. In both panels the curves are labeled by the $[\mathrm{Fe} / \mathrm{H}]$ value. The solid horizontal lines represent the observed value of $\Delta H_{\mathrm{HB}}^{\mathrm{TO}}$ for Terzan 5 , and the dotted lines represent the estimated range of uncertainty.

the metallicity. However, it can be seen that the uncertainty ranges are significantly different for the two calibration methods. For an evolving HB magnitude, the best-fit ages are $15 \mathrm{Gyr}$ for solar metallicity and $10 \mathrm{Gyr}$ for supersolar metallicity, with lower limits of 8 and 7 Gyr, respectively. The flattening of the calibration curves in this case precludes placing upper limits on the ages for the estimated $0.2 \mathrm{mag}$ precision of $\Delta H_{\mathrm{HB}}^{\mathrm{TO}}$. The limits are tightened significantly for a fixed HB magnitude, where the best-fit ages are $14_{-4}^{+5} \mathrm{Gyr}$ for solar metallicity and $11_{-3}^{+4}$ Gyr for supersolar metallicity.

We note that Ortolani et al. (2001) have independently determined an age for Terzan 5 by fitting isochrones to a CMD determined from their NIC1 observations of a field $30^{\prime \prime}$ from the cluster center. They find an identical offset of $4.3 \pm 0.2$ mag between the HB and the MSTO, at F110W, to what we find at $H$, from which they infer an age of $14 \pm 3$ Gyr, using newly computed isochrones in F110W-F160W $H S T$ filter system. They assume a fixed ZAHB magnitude, taken to be that of a star of initial mass of $1 M_{\odot}$. They also adopt solar metallicity. Thus, they have treated the case represented by the lower curve in the lower panel of Figure 7, for which our best-fit age is also 14 Gyr. However, their computed values of $\Delta \mathrm{F} 110 \mathrm{~W}_{\mathrm{HB}}^{\mathrm{TO}}$ have a steeper dependence on age than do the values for $\Delta H_{\mathrm{HB}}^{\mathrm{TO}}$ that we derived from the Girardi et al. (2000) isophotes, allowing Ortolani et al. (2001) to place somewhat tighter limits on the determined age ( \pm 3 vs. $\pm 4.5 \mathrm{Gyr}$ ). This evidently results from a difference in the behavior of the isochrone sets. The good agreement between the best-fit values for the age of Terzan 5 (assuming solar metallicity), obtained here and by Ortolani et al. (2001), is encouraging. However, the assumption of a fixed HB magnitude, when not using RR Lyrae stars to define the HB, should be investigated further.

\section{CLUSTER STRUCTURE}

Trager, King, \& Djorgovski (1995) have studied the spatial structure of Terzan 5 by determining the surface-brightness profile from an $I$-band CCD image. They determined a core radius value of $r_{c}=10.7$ and a half-mass radius of $r_{h}=55^{\prime \prime}$. Since small numbers of individual bright giants dominate the surface-brightness distribution, especially in the $I$ band, star-count profiles generally provide a better means of determining the cluster structure (see, e.g., Sosin \& King 1997).

We have constructed a surface-density profile for Terzan 5 , using star counts from the central and offset NIC2 fields (see Fig. 1). It is necessary to include the offset field, since the cluster core fills the central field. We used the F187W star counts, since this is the only broadband filter common between these two fields. Since our exposures for the offset field are not dithered, it was not feasible to perform PSFsubtraction photometry for these frames. Instead, we used simple aperture photometry and computed a magnitude offset between the central and offset fields from the stars in the overlap region. Since the depth of the star counts varies with distance from the cluster center, we adopted a uniform limiting magnitude for stars to be included in the profile determination that corresponds to 2 mag below the mean HB, i.e., $\mathrm{F} 187 \mathrm{~W}=15.9$. Figure 5 indicates that the central field is fairly complete to this limit.

We used iterative centroiding, in a circular aperture with an initial radius equal to the half width of the NIC2 field (9".6), to determine the location of the cluster center. As the centroid shifted from the starting position at the frame center, we reduced the size of the aperture to keep it entirely within the central NIC2 field at each iteration. The iteratively determined centroid was offset by 0.77 from the frame center. Trials with a range of values of the limiting magnitude and aperture size indicate that the uncertainty in the location of the center is on the order of $1^{\prime \prime}$. Our determined center position corresponds to the equatorial coordinates (J2000) $\alpha=17^{\mathrm{h}} 48^{\mathrm{m}} 04^{\mathrm{s}} .8$ and $\delta=-24^{\circ} 46^{\prime} 45^{\prime \prime}$. Table $1 \mathrm{com}-$ pares this position with previous determinations. There is particularly good agreement between the present center determination and those of Djorgovski \& Meylan (1993, after correction; see Table 1 note) and Fruchter \& Goss (2000). The latter is the centroid of the unresolved radio flux from the core, which they suggest arises from an unresolved MSP population. The close agreement between this radio center and our present optical center is striking.

We binned the star counts into a central disk of radius 0 ".75 and nine concentric, logarithmically spaced circular annuli that extend to $29^{\prime \prime}$ from the cluster center; the annuli are incomplete beyond $9^{\prime \prime}$. The surface density in each annulus is computed by dividing the number of stars in that annulus by the area of the annulus that lies within the combined NIC2 central and offset fields. Figure 8 shows the 
TABLE

Comparison of Cluster Center Determinations

\begin{tabular}{|c|c|c|c|}
\hline Study & $\alpha(\mathrm{J} 2000)$ & $\delta(\mathrm{J} 2000)$ & $\begin{array}{c}\Delta^{\mathrm{a}} \\
(\operatorname{arcsec})\end{array}$ \\
\hline Terzan 1971. & 174804.6 & -244650.9 & 6.7 \\
\hline Djorgovski \& Meylan $1993^{\mathrm{b}} \ldots . .$. & 174804.9 & -244645 & 1.1 \\
\hline Picard \& Johnston 1995 ............ & 174804.6 & -244648.5 & 4.7 \\
\hline Fruchter \& Goss $2000 \ldots \ldots \ldots \ldots \ldots$ & 174804.9 & -244644.7 & 1.3 \\
\hline Present study & 174804.8 & -244645.0 & . \\
\hline
\end{tabular}

NotE.-Units of right ascension are hours, minutes, and seconds, and units of declination are degrees, arcminutes, and arcseconds. All positions are given with the same precision as in the original source.

a Total offset relative to present study.

b A misprint in the $\delta$ minutes value has been corrected per private communication with S. Djorgovski.

resulting surface-density profile. We have included the Trager et al. (1995) surface-brightness profile for comparison, ${ }^{5}$ with a shift applied to it so that the two profiles agree near $r=10^{\prime \prime}$. It can be seen that both profiles shown in Figure 8 have relatively flat cores, with a somewhat smaller core radius for our surface-density profile.

In order to determine the core radius in a manner independent from the adopted binning, we employed the maximum likelihood method outlined by Sosin \& King (1997). In this approach, a surface density profile function is fitted to the star position data directly, without resort to radial binning. The likelihood for the observation of a particular

${ }^{5}$ The Trager et al. (1995) data set includes three spectral bands, $B, R$, and $I$. The $I$ and $R$ data cover the entire profile, while the $B$ data cover only the outer halo. Since the $I$ and $R$ data each tightly define a profile that is slightly different from that defined by the other band, we chose to include only their $B$ and $I$ data in this comparison.

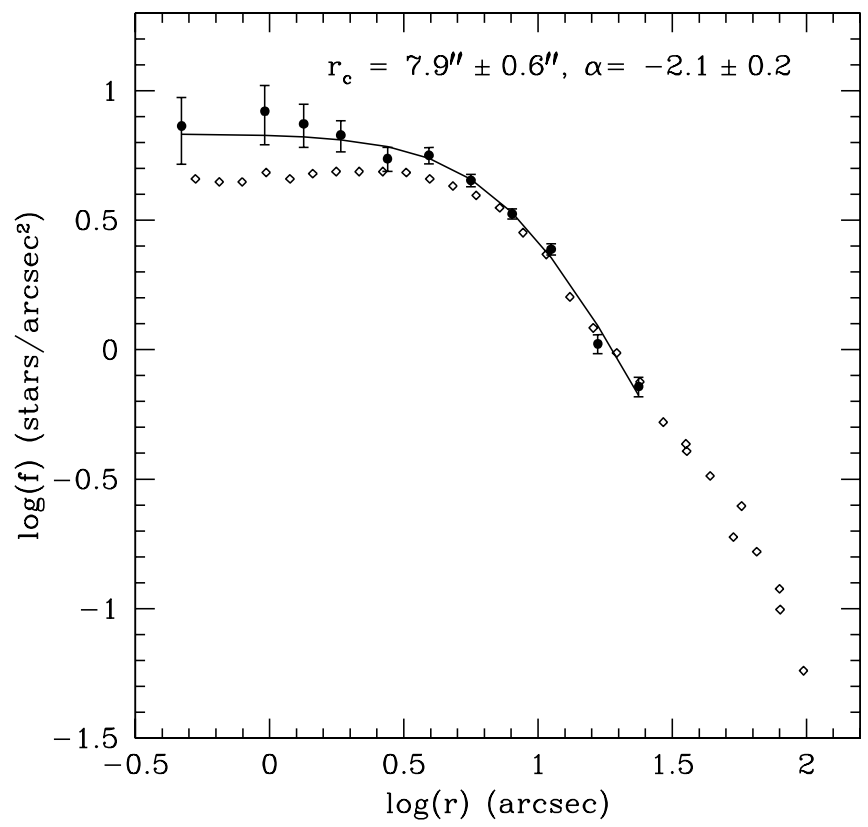

FIG. 8.- Surface-density profile from F187W star counts ( filled circles and error bars) compared with surface brightness profile from Trager et al. (1995) (open diamonds). The solid line is a maximum likelihood fit of eq. (4) to the unbinned $\mathrm{F} 187 \mathrm{~W}$ star counts. set of stellar radial position values $\left(r_{i}\right)$ is given by

$$
L(\boldsymbol{a})=\prod_{i=1}^{N} f\left(r_{i}, \boldsymbol{a}\right)
$$

where $f$ represents a parameterized form for the surface density profile (in units of stars per unit area) and $\boldsymbol{a}$ represents a set of parameters on which $f$ depends. It is convenient to work with $\log L$, which converts the product in equation (3) to a sum. Rather than compute $\log L$ over a grid in parameter space, to locate the point of maximum likelihood, we found it more convenient to use an optimization routine. We used an implementation of Powell's method from Numerical Recipes (Press et al. 1992, $§ 10.5$ ), which does not require the computation of derivatives of the likelihood function with respect to the parameters. For estimating the confidence ranges for the parameter estimates, we used the "bootstrap" method, i.e., random resampling of the star list with replacement (Efron 1982; Press et al. 1992, § 15.6). We implemented this by drawing 1000 random resamples and determining the maximum likelihood values of the parameters for each resample. We verified that the distributions of the parameter values determined from the set of resamples are closely approximated by Gaussians, with means equal to the best-fit parameter values for the original sample. The standard deviations of these distributions provide robust estimates for the uncertainty of the best-fit parameter values.

Following Lugger, Cohn, \& Grindlay (1995), we used the following modified power-law form for the azimuthally symmetric surface density distribution,

$$
f(r)=f_{0}\left[1+\left(\frac{r}{r_{0}}\right)^{2}\right]^{\alpha / 2} .
$$

Here $r_{0}$ is a radial scale related to the core radius and $\alpha$ is the power-law index of the outer part of the profile. The normalization parameter $f_{0}$ is determined by the condition that the integral of $f(r)$ over the area in which the stars are located remains constant as the values of the other two parameters are varied. For the usual definition of the core radius as the point at which the surface density decreases to half of its central value, $r_{c}=\left(2^{-2 / \alpha}-1\right)^{1 / 2} r_{0}$. A power-law slope of $\alpha=-2$ corresponds to an "analytic King model" (King 1962). Lugger et al. (1995) have shown that equation (4) with $\alpha=-1.8$ provides an excellent fit to an actual King (1966) model with a central concentration of $c=2.0$, for all but the outer halo. As a consistency check, we performed a least-squares fit of equation (4) to the Trager et al. (1995) profile. We find $r_{c}=10^{\prime \prime} \cdot 2$ and $\alpha=-1.9$. This core radius value agrees with their value of 10.7 to within $5 \%$; the power-law slope is consistent with a King model.

Our maximum likelihood fit of equation (4) to the NIC2 star counts produces $r_{c}=7 "$. $9 \pm 0$ ". 6 and $\alpha=-2.1 \pm 0.2$. It can be seen in Figure 8 that this form provides an excellent fit. The core radius is about $25 \%$ smaller than that found by Trager et al. (1995), while the power-law slope is similar to that produced by our fit to their profile. Interestingly, in a preliminary report of their profile fits, Trager, Djorgovski, \& King (1993) found a core radius of 7"! 9 , in agreement with our value. The slope value of $\alpha \approx-2$ again indicates consistency with a King model.

To further explore the limits on the central concentration of Terzan 5 that can be obtained from model fitting to the 
combined profile shown in Figure 8, we have overplotted a set of King models with a range of $c$ from 1.75 to 2.25 , as shown in Figure 9. Rather than formally fitting the profiles, we simply adopted the core radius and central surface density values from our maximum likelihood fits of the modified power-law profile. It can be seen in Figure 9 that the $c=2.0 \mathrm{King}$ model provides the best overall fit to the outer part of the profile as determined by Trager et al. (1995). Given the challenge of determining the surface brightness profile at large distance from the cluster center, with the high background of the Galactic bulge and variable extinction, we do not view this as a rigorous determination of the central concentration of Terzan 5. Rather, we see it as a demonstration that a relatively high central concentration of $c \approx 2$ is certainly plausible.

For our adopted normalization of the profiles near $r=10^{\prime \prime}$, the central surface density is $60 \%$ higher for the NIC2 profile than for that of Trager et al. (1995). The combination of the higher surface density and the smaller core radius implies that the central density is about twice as large as the value of $\rho_{0}=10^{5.76} M_{\odot} \mathrm{pc}^{-3}$ determined by Djorgovski (1993), i.e., $\rho_{0} \approx 10^{6.0} M_{\odot} \mathrm{pc}^{-3}$. This puts Terzan 5 at the top of the central density distribution for all clusters, although the tabulated values for collapsed-core clusters are more properly viewed as lower limits. Following Djorgovski (1993), we may estimate the central relaxation time of Terzan 5 , by applying the scaling relation, $t_{\mathrm{rc}} \propto \rho_{0}^{0.5} r_{c}^{3}$, to his estimate. The result is $t_{\mathrm{rc}}=4 \times 10^{7} \mathrm{yr}$, which is at about the 30th percentile of the distribution for 124 clusters from Djorgovski (1993). Of the clusters with shorter central relaxation times than Terzan 5, only about one third have apparently "normal" cores, i.e., a central structure that is well fitted by a King model, like that of Terzan 5 . Thus, Terzan 5 has both an extremely high central density and an unusually short relaxation time for a cluster with a noncollapsed core.

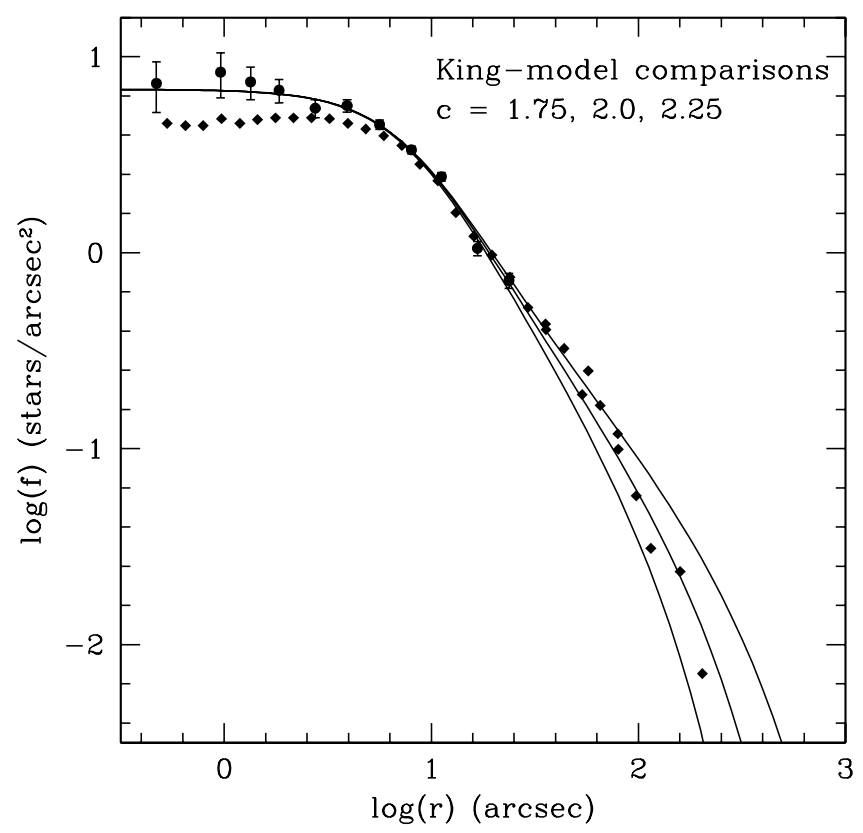

FIG. 9.-Combined surface-density profile (as described in Fig. 8) with King model overlays. The curves correspond to the indicated $c$-values from left to right. The core radius and central density are taken from the maximum likelihood fit shown in Fig. 8. Note that the best match is obtained for concentration parameter in the range $2.0 \lesssim c \lesssim 2.25$.

\section{DISCUSSION}

Using NIC2 imaging of the central $19^{\prime \prime} \times 19^{\prime \prime}$ region of Terzan 5 and NICl imaging of a field $30^{\prime \prime}$ from the cluster center, we have constructed color-magnitude diagrams and produced new measurements of the cluster reddening, distance, and metallicity. We have also determined the surfacedensity profile and performed a maximum likelihood fit to measure the core radius and halo slope. Our results underscore the extraordinary nature of this cluster.

The result that the reddening is somewhat smaller than found by Ortolani et al. (1996) places the cluster at a greater distance than the strikingly small value of $5.6 \mathrm{kpc}$ that they found. This new reddening, together with our measurement of the $J$-band magnitude of the horizontal branch, place Terzan 5 at the $60 \%$ larger distance of $8.7 \mathrm{kpc}$ and thus within less than $1 \mathrm{kpc}$ of the Galactic center. Our distance measurement is consistent with the range of 7-9 kpc produced by most studies prior to Ortolani et al. (1996).

The probable detection of the red giant branch bump, about 1 mag below the horizontal branch, indicates that the metallicity of Terzan 5 is at least solar and possibly somewhat higher $\left([\mathrm{Fe} / \mathrm{H}]_{785}=+0.26\right)$. This places Terzan 5 at the very top of the metallicity distribution of all globular clusters. This suggests that Terzan 5 was formed in the vicinity of its present location near the Galactic center.

The detection of the main-sequence turnoff has allowed us to estimate the cluster age using the magnitude difference between the turnoff and the horizontal branch. Our result for the best-fit age-14 Gyr for an assumed solar metallicity and a fixed HB magnitude - is the same as that recently obtained independently by Ortolani et al. (2001) from a separate NICMOS data set. However, our derived uncertainty in the age is somewhat larger than they obtained, owing to differences in the isochrone sets used. Even with their tighter age precision of $\pm 3 \mathrm{Gyr}$, it is only marginally possible to distinguish between "young" ( 10 Gyr) and "old" ( 15 Gyr) ages for Terzan 5 . We also note the role of metallicity in the age determination; Figure 7 indicates that the inferred age may be reduced by 3-5 Gyr if supersolar metallicity were adopted. Given the extraordinarily high metallicity of Terzan 5, a tighter limit on the cluster age would have important implications for the chemical history of the Galactic center region, particularly if the age turned out to be fairly old.

Improvements in the age estimate could be achieved by a combination of (1) NIC1 mosaic imaging of the central region to improve the photometric precision and depth, (2) additional NICMOS imaging at about $0.5-1^{\prime}$ from the cluster center, to improve the statistics of the deep CMD, (3) NICMOS observations of more near-infrared standard stars, to improve the determination of the magnitude system transformations, (4) additional study of infrared isochrones to better fit the observations of high-metallicity systems, and (5) a tighter limit on the metallicity of Terzan 5.

Our maximum likelihood fit of a modified power-law form to the surface-density profile within 0.5 of the cluster center has produced a new core radius measurement of $r_{c}=7 "$. 9 , which is somewhat smaller than the previous determination by Trager et al. (1995). Nevertheless, our results underscore the fact that Terzan 5 has a resolved core and is well fitted by a King-model profile. Given the relatively short central relaxation time of $t_{\mathrm{rc}}=4 \times 10^{7} \mathrm{yr}$, it is interesting to ask whether Terzan 5 should have already 
undergone core collapse. For a single-mass cluster, the core collapse time is formally about 300 times longer than the central relaxation time (Cohn 1980). This would suggest that Terzan 5 may be just now at the brink of core collapse (see also Cohn \& Hut 1984). Furthermore, Meylan \& Heggie (1997) have argued that any cluster with central concentration $c \gtrsim 2$ should be considered to be either on the brink of core collapse or just past it.

While the concentration parameter of Terzan 5 has not been directly determined, given the difficulty of determining the tidal radius of this highly obscured cluster, our comparisons with King models suggest that $c \approx 2$. Given the estimated half-mass radius of Terzan 5 from Trager et al. (1995), the ratio of core to half-mass radius is $r_{c} / r_{h}=0.15$. Grabhorn et al. (1992) have shown that this ratio has a maximum value of about $0.01-0.04$ in post-collapse dynamical models for clusters. This suggests that the core of Terzan 5 may still be supported in a precollapse state by energy release ("heating") from primordial binaries that interact dynamically with other stars. Alternatively, Terzan 5 may be in a post-collapse state with a "superexpanded" core, produced by the action of a residual population of primordial binaries. Such binaries were not considered by Grabhorn et al. (1992).

If Terzan 5 is indeed near the point of core collapse, we might expect to see enhanced binary activity in its core. Ferraro et al. (1999b) have interpreted the huge blue straggler population that they detected in the high-density, noncollapsed core of M80 as evidence of vigorous binary heating, which is temporarily staving off imminent collapse. Specifically, they propose that the blue stragglers are the result of the hardening of both primordial binaries and binaries produced by tidal capture and three-body interactions. The vast majority of blue stragglers present in Terzan 5 should lie in the central NIC2 field of our data set, given the extreme central concentration of blue straggler populations in dynamically evolved clusters. The likely eclipsing blue straggler identified by Edmonds et al. (2001) lies at about 8 "from the cluster center. Highly dithered NIC1 photometry of the central region may aid in detecting additional blue stragglers in the core, although distinguishing between blue stragglers and photometric artifacts in this crowded central region could prove challenging. Ground-based adaptive optics observations with the Hokupa'a system on Gemini may well prove superior to NICMOS for probing the core of Terzan 5 , given the large telescope aperture, the 0 ".02 pixel size of the imager, the $20^{\prime \prime}$ field of view, and the 0 ". 07 or better expected FWHM of the images under good seeing conditions.

Multiwavelength studies of Terzan 5, including additional searches for millisecond pulsars and X-ray sources, will certainly be useful for assessing the level of stellar collisional activity in the core. Our group has recently obtained a deep Chandra observation of Terzan 5. However, the LMXB was in a high state during this observation, which may preclude the detection of a large population of faint $\mathrm{X}$ ray sources in the central region. In any case, the X-ray spectral observations should provide a measurement of the hydrogen column density toward Terzan 5, and thus an independent estimate of the obscuration. This will provide a check on our new distance estimate. Our NICMOS study underscores the extraordinary physical conditions present in the core of Terzan 5. Thus, it remains of great interest to test the prediction that this region is a factory for the production of substantial numbers of interacting binaries.

We acknowledge helpful conversations with Charles Bailyn, Adrienne Cool, Con Deliyannis, and Ata Sarajedini. We also appreciate the insightful review by the anonymous referee, which improved the manuscript. This research was supported in part by NASA grants GO-07889.01-96A to Harvard University and GO-07889.03-96A to Indiana University.
Armandroff, T., \& Zinn, R. 1988, AJ, 96, 92

Barbuy, B., Renzini, A., Ortolani, S., Bica, E., \& Guarnieri, M. D. 1999, A\&A, 341, 539

Bedin, L. R., Piotto, G., Zoccali, M., Stetson, P. B., Saviane, I., Cassisi, S., \& Bono, G. 2000, A\&A, 363, 159

Bica, E., Clariá, J. J., Piatti, A. E., \& Bonatto, C. 1998, A\&AS, 131, 483

Carney, B. W., Fulbright, J. P., Terndrup, D. M., Suntzeff, N. B., \& Walker, A. R. 1995, AJ, 110,1674

Chaboyer, B., Demarque, P., \& Sarajedini, A. 1996, ApJ, 459, 558

Coelho, P., Barbuy, B., Perrin, M.-N., Idiart, T., Schiavon, R. P., Ortolani, S. \& Bica, E. 2001, A\&A, 376, 136

Cohen, J. G., Gratton, R. G., Behr, B. B., \& Carretta, E. 1999, ApJ, 523, 739

Cohn, H. 1980, ApJ, 242, 765

Cohn, H., \& Hut, P. 1984, ApJ, 277, L45

Cool, A. M., Grindlay, J. E., Cohn, H. N., Lugger, P. M., \& Slavin, S. D. 1995, ApJ, 439, 695

Davidge, T. J. 2000, ApJS, 126, 105

2001, AJ, 121,3100

Davidge, T. J., \& Courteau, S. 1999, AJ, 117, 1297

Djorgovski, S. 1993, in ASP Conf. Proc. 50, Structure and Dynamics of Globular Clusters, ed. S. G. Djorgovski \& G. Meylan (San Francissco: ASP), 373

Djorgovski, S., \& Meylan, G. 1993, in ASP Conf. Proc. 50, Structure and Dynamics of Globular Clusters, ed. S. G. Djorgovski \& G. Meylan (San Francissco: ASP), 325

Edmonds, P. D., Grindlay, J. E., Cohn, H., and Lugger, P. 2001, ApJ, 547, 829

Efron, B. 1982, The Jackknife, the Bootstrap, and Other Resampling Plans (Philadelphia: SIAM)

Feltzing, S., \& Gilmore, G. 2000, A\&A, 355, 949

Ferraro, F. R., Messineo, M., Fusi Pecci, F., De Palo, M. A., Straniero, O., Chieffi, A., \& Limongi, M. 1999a, AJ, 118, 1738

\section{EFERENCES}

Ferraro, F. R., Paltrinieri, B., Rood, R. T., \& Dorman, B. 1999b, ApJ, 522, 983

Frogel, J. A., Tiede, G. P \& Kuchinski, L. E. 1999, AJ, 117, 2296

Frogel, J. A., \& Whitford, A. E. 1987, ApJ, 320, 199

Fruchter, A. S., \& Goss, W. M. 1995, J. Astrophys. Astron., 16, 245 2000, ApJ, 536, 865

Fusi Pecci, F., Ferraro, F. R., Crocker, D. A., Rood, R. T., \& Buonanno, R. 1990, A\&A, 238, 95

Girardi, L., Bressan, A., Bertelli, G., \& Chiosi, C. 2000, A\&AS, 141, 371

Girardi, L., \& Salaris, M. 2001, MNRAS, 323, 109

Grabhorn, R. P., Cohn, H. N., Lugger, P. M., \& Murphy, B. W. 1992, ApJ 392,86

Harris, W. E. 1996, AJ, 112, 1487

Hook, R. N., \& Fruchter, A. S. 1997, in ASP Conf. Ser. 125, Astronomical Data Analysis Software and Systems VI, ed. G. Hunt \& H. E. Payne (San Francisco: ASP), 147

Hook, R. N., Pirzkal, N., \& Fruchter, A. S. 1999, in ASP Conf. Ser.

172, Astronomical Data Analysis Software and Systems VIII, ed. D. Mehringer, R. Plante, \& D. Roberts (San Francisco: ASP), 337

Iben, I., Jr. 1968, Nature, 220, 143

Johnston, H., Verbunt, F., \& Hasinger, G. 1995, A\&A, 298, L21

King, I. R. 1962, AJ, 67, 471 1966, AJ, 71, 64

Lugger, P. M., Cohn, H. N., \& Grindlay, J. E. 1995, ApJ, 439, 191

Lyne, A. G., Mankelow, S. H., Bell, J. F., \& Manchester, R. N. 2000 MNRAS, 316, 491

Malkan, M. A. 1982, in IAU Colloq. 68, Astrophysical Parameters for Globular clusters, ed. A. G. D. Philip \& D. S. Hayes (New York: Springer), 533

McNamara, D. H., Madsen, J. B., Barnes, J., \& Ericksen, B. F. 2000, PASP 112,202

Meylan, G., \& Heggie, D. G. 1997, Astron. Astrophys. Rev., 8,

Mollá, M., Ferrini, F., \& Gozzi, G. 2000, MNRAS, 316, 345 
Nice, D. J., \& Thorsett, S. E. 1992, ApJ, 397, 249

Ortolani, S., Barbuy, B., \& Bica, E. 1996, A\&A, 308, 733

Ortolani, S., Barbuy, B., Bica, E., Renzini, A., Aoccali, M., Rich, R. M., \& Cassisi, S. 2001, A\&\&, 376, 878

Picard, A., \& Johnston, H. M. 1995, A\&AS, 112, 89

Press, W. H., Teukolsky, S. A., Vetterling, W. T., \& Flannery, B. P. 1992,

Numerical Recipes in C (2d ed.; Cambridge: Cambridge Univ. Press)

Ramírez, S. V., Stephens, A. W., Frogel, J. A., \& DePoy, D. L. 2000, AJ, 120,833

Rich, R. M., et al. 1997, ApJ, 484, L25

Rieke, G. H., \& Lebofsky, M. J. 1985, ApJ, 288, 618

Rosenberg, A., Saviane, I., Piotto, G., \& Aparicio, A. 1999, AJ, 118, 2306
Schulte-Ladbeck, R. E., Hopp, U., Greggio, L., \& Crone, M. M. 1999, AJ, 118,2705

Sosin, C., \& King, I. R. 1997, AJ, 113, 1328

Stetson, P. B. 1987, PASP, 99, 191

Stetson, P. B., \& Harris, W. E. 1988, AJ, 96, 909

Terzan, A. 1971, A\&A, 12, 477

Trager, S. C., Djorgovski, S., \& King, I. R. 1993, in ASP Conf. Proc. 50 Structure and Dynamics of Globular Clusters, ed. S. G. Djorgovski \& G. Meylan (San Francisco: ASP), 347

Trager, S. C., King, I. R., \& Djorgovski, S. 1995, AJ, 109, 218

Verbunt, F., \& Hut, P. 1987, IAU Symp. 125, The Origin and Evolution of Neutron Stars, ed. D. J. Helfand \& J.-H. Huang (Dordrecht: Reidel), 187 Zinn, R. 1985, ApJ, 293, 424 (Z85) 